\title{
Unusual Coexistence of Pulmonary Tuberculosis and Hydatid Disease
}

\author{
${ }^{1}$ Deepthi Laldayal, ${ }^{2}$ Ketaki Utpat, ${ }^{3}$ Jyotsna M Joshi
}

\begin{abstract}
Tuberculosis and hydatidosis are both infectious diseases commonly involving the pulmonary system and exhibiting a wide spectrum of clinical manifestations of varying severity. The simultaneous occurrence of pulmonary tuberculosis and hydatid disease is extremely rare, reported sporadically in the literature. We herein report two cases where this rare coexistence was proven after radiological features aroused suspicion. The first patient was a young female with a short history of fever and cough. She had miliary mottling and a cystic lesion on imaging. Second patient was an elderly lady with a long-standing cough, dyspnea, and hemoptysis. She had multiple cystic and cavitatory lesions on imaging. Both of them were managed with medical therapy successfully. The possibility of hydatid tuberculosis coinfection should be kept in mind in endemic areas in the relevant clinical scenarios.
\end{abstract}

Keywords: Coinfection, Hydatid disease, Pulmonary tuberculosis.

How to cite this article: Laldayal D, Utpat K, Joshi JM. Unusual Coexistence of Pulmonary Tuberculosis and Hydatid Disease. J Postgrad Med Edu Res 2018;52(1):36-39.

Source of support: Nil

Conflict of interest: None

\section{INTRODUCTION}

Tuberculosis and pulmonary hydatidosis are two endemic diseases in our country. Tuberculosis has evolved itself as the most common infectious disease in India with myriad of presentations. Sixty-five million people are infected globally with hydatid disease and it is considered a major health problem in India ${ }^{1}$ too. The two diseases, tuberculosis and hydatid disease, occurring simultaneously in an individual, however, is an extremely rare incident. The primary infection in both diseases is believed to occur in childhood, though the modes of infection are different. Their coinfection in a 5-year-old has been reported ${ }^{2}$ in literature. The earliest report of this

\footnotetext{
${ }^{1}$ Junior Resident, ${ }^{2}$ Assistant Professor, ${ }^{3}$ Professor and Head

${ }^{1-3}$ Department of Pulmonary Medicine, Topiwala National Medical College \& B.Y.L. Nair Ch. Hospital, Mumbai, Maharashtra, India

Corresponding Author: Jyotsna M Joshi, Professor and Head Department of Pulmonary Medicine, Topiwala National Medical College \& B.Y.L. Nair Ch. Hospital, Mumbai, Maharashtra, India Phone: +912223027642, e-mail: drjoshijm@gmail.com
}

coinfection was from India in $1986 .{ }^{3}$ There is paucity of literature about the effect of one infection on other, the associated complications, and treatment. As reported in other parasitic infestations, a change in immune system mechanisms in Echinococcosis may cause reactivation of primary tuberculous infection. ${ }^{4}$ Unconventional clinical and radiological presentations in such coinfections pose a diagnostic and therapeutic challenge to physicians.

\section{CASE REPORTS}

\section{Case 1}

A 20-year-old woman presented with 20 days history of fever, cough with mucoid expectoration, Modified Medical Research Council grade I dyspnea, and left-sided chest pain. On general examination, she had a $1 \times 1 \mathrm{~cm}$ supraclavicular lymph node. Examination of respiratory system was unremarkable. Her chest radiograph (CXR) revealed bilateral military opacities and left midzone cystic lesion (Fig. 1). High-resolution computed tomography (HRCT) thorax showed multiple randomly distributed nodules, necrotic mediastinal and hilar lymphadenopathy, and well-defined cystic lesion in lingular segment of the left upper lobe. Sputum examination by Gene Xpert assay did not pick up Mycobacterium tuberculosis (MTB). Fine needle aspiration cytology of supraclavicular lymph node showed granulomatous inflammation. Indirect hemagglutination (IHA) against Echinococcus granulosus was negative. Provisional diagnosis of military tuberculosis (TB) with left upper lobe hydatid cyst was kept in view of her clinicoradiological presentation and patient was empirically started on antituberculous therapy (ATT). Ultrasonography-guided aspiration of cyst showed transudative clear fluid with no evidence of membranes or scolices. Postaspiration chest CXR showed water lily sign. Repeat sputum examination by Gene Xpert assay picked up MTB, with rifampicin sensitivity. There was defervescence of fever with ATT and she was discharged after adding oral tablet Albendazole $15 \mathrm{mg} / \mathrm{kg}$ weight. She was not offered surgery at that point of time in view of the prevalence of active tuberculous infection. On completion of ATT, the patient was asymptomatic with reduction in the size of the cyst radiologically (Fig. 2). She was continued on Albendazole therapy and is currently under regular follow-up. 


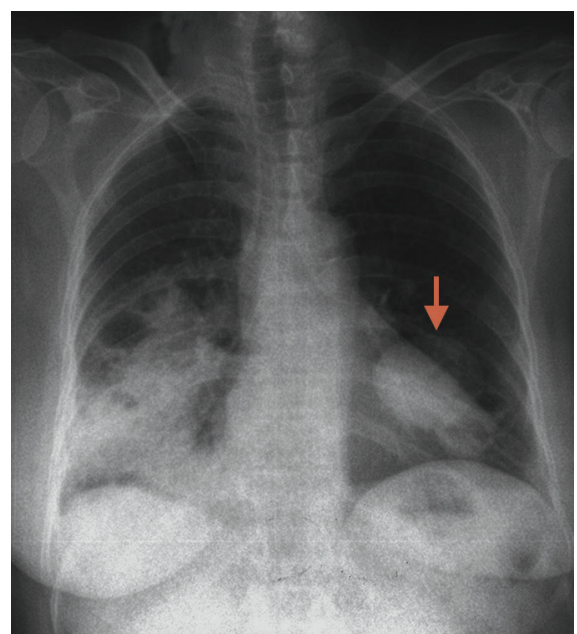

Fig. 1: Chest X-ray showing multiple cystic lesions with left lower zone cyst highlighted

\section{Case 2}

A 60-year-old female, with no history of addictions, presented with a 20-year history of dyspnea, cough with mucoid expectoration, and multiple episodes of streaky hemoptysis managed with symptomatic treatment in peripheral centers. An increase in cough and dyspnea in the preceding 4 months led to further evaluation with CXR (Fig. 3), showing multiple bilateral cystic opacities with some cysts showing air-fluid levels. Physical examination revealed bilateral crackles and rhonchi. Allergic bronchopulmonary aspergillosis vs hydatid disease was the differential diagnoses kept in view of her long-standing history and radiological picture. Specific IgE against Aspergillus fumigatus was negative and IHA against $E$. granulosus was positive. Further HRCT thorax revealed multiple cystic lesions in bilateral lower lobes, with one cyst showing meniscus sign along with cavitation and right lower lobe consolidation, consistent with hydatid disease with multifocal involvement. Sputum examination by Gene

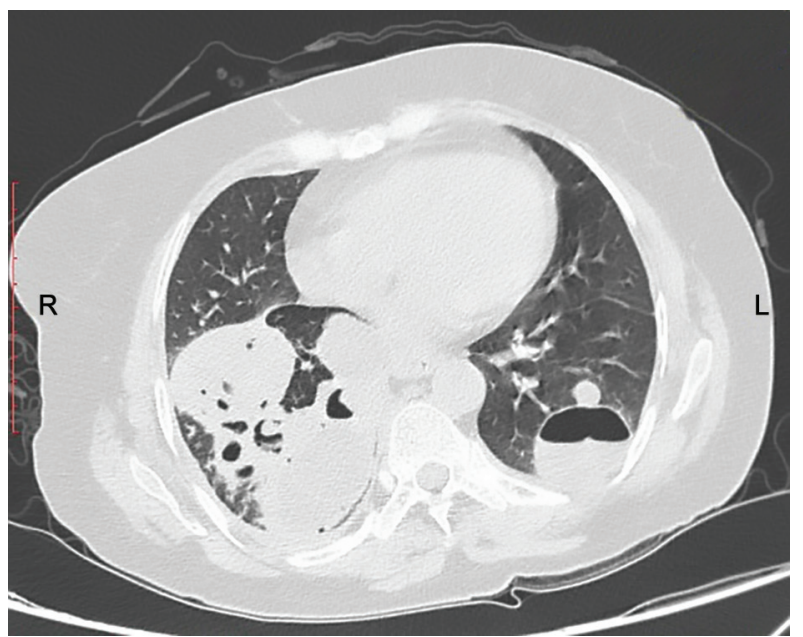

Fig. 3: Computed tomography thorax showing right lower lobe consolidation and left lower lobe cyst showing water lily sign post-tap

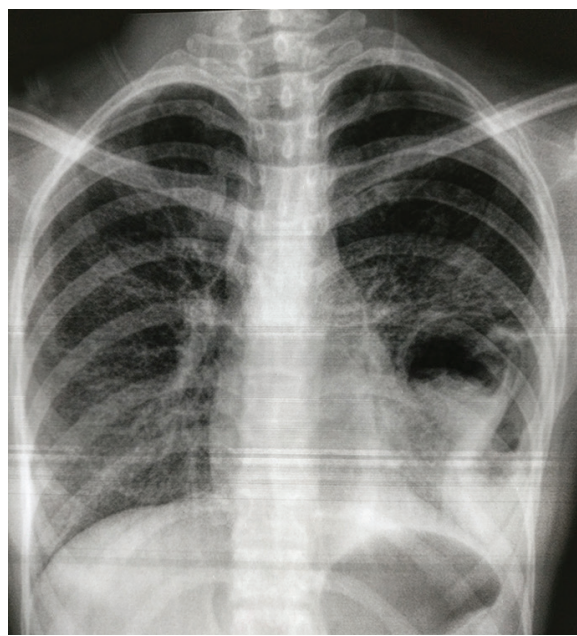

Fig. 2: Post-tap chest X-ray showing water lily sign highlighted

Xpert assay did not detect MTB. Flexible bronchoscopy showed no evidence of membranes, but secretions were seen in right lower lobe bronchus. Bronchial washing analysis for gene Xpert assay detected mycobacterium tuberculosis and reavealed a sensitivity to Rifampicin. The computed tomography (CT)-guided aspiration of left cyst showed clear fluid with no scolices. Post-tap, the cyst showed water lily sign on CXR (Fig. 4) and CT (Fig. 5). Spirometry showed an obstructive pattern with poor bronchodilator reversibility. Patient was started on ATT, albendazole, and bronchodilator therapy. On follow-up, patient reported significant improvement in symptoms with no further episodes of hemoptysis.

\section{DISCUSSION}

Through the centuries, TB has remained a ubiquitous infectious disease. Other coinfections with TB have been variably reported in literature. Hydatid disease is one such coinfection, which is uncommonly reported and

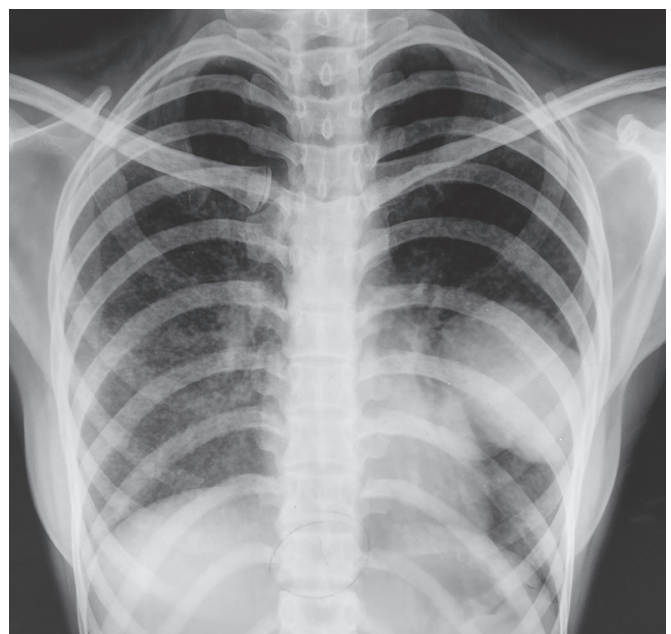

Fig. 4: Chest X-ray showing miliary opacities and left lower zone cystic lesion 


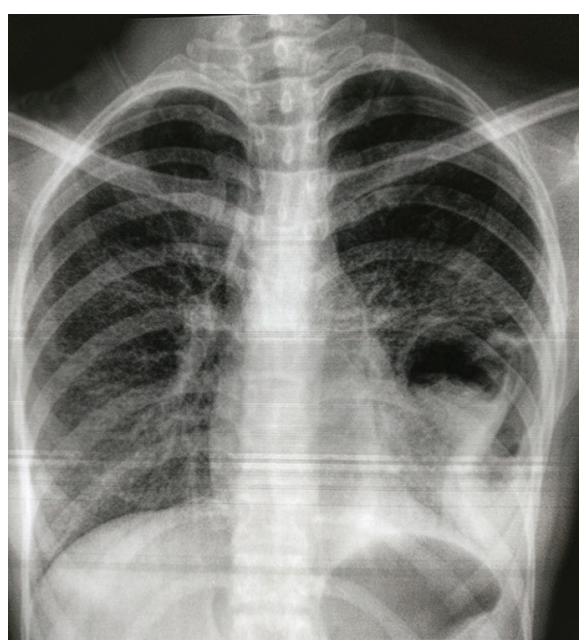

Fig. 5: Chest $\mathrm{X}$-ray showing clearing of miliary opacities and decrease in size

studied. Hydatid disease is significantly prevalent in the Indian subcontinent, especially in the rural regions. Humans acquire infection through close contact with dogs, the definitive host, ${ }^{5}$ or by consumption of contaminated water and vegetables. Most common site of involvement is liver followed by lungs. Poor hygiene and low socioeconomic status contribute significantly to acquisition of both these diseases. Helminthic infections cause a shift in immunological balance by suppressing the Th1 immune response and elevation of Th2 immune response. This, in turn, renders the host susceptible to a multitude of viral and bacterial infections. A study on immunological aspects concerning this coinfection in China ${ }^{6}$ showed a suppression of Th1 immunoresponse as indicated by conversion of a positive tuberculin test to negative in long-standing untreated patients with Echinococcosis.

Hydatid cysts can be bilateral in $20 \%$, multiple in $30 \%$, and involves the right side and lower lobes in $60 \%$ of cases. ${ }^{7}$ No age or sex predilection could be seen. Clinical picture may vary depending on the site of cyst, size of cyst, and whether the cyst is ruptured or intact. Unruptured cysts are usually asymptomatic and incidentally detected during evaluation for any other cause. Symptoms in hydatid cyst result from a large cyst exerting mechanical effects on adjacent structures or due to complications resulting from a ruptured cyst. Cyst may rupture into the bronchus, pleural cavity, or biliary tree. In these situations, patients may present with symptoms of cough with salty sputum, hemoptysis, chest pain, or dyspnea. Occasionally, there maybe expectoration of the germinal membrane or hooklets referred to as hydatoptysis due to perforation of cysts into a bronchiole. Moreover, the clinical manifestations of pulmonary TB are also heterogeneous and nonspecific, largely dependent on the bacillary load, the host immunity, and the extent of organ involvement. Thus, in both conditions, symptomatology may be similar leading to diagnostic dilemma. Our first patient is a young female with a short history. She had an intact cyst and hence, her hydatid disease was subclinical. It was picked up as an incidental finding during the workup done for her suspected TB. The second case was an elderly female with a long history and positive serology for hydatid disease. This could be explained by her ruptured cysts, which was confirmed radiologically. Right lower lobe consolidation due to TB might have led to acute worsening of her symptoms. Both patients denied any close contact with dogs. To conclude, the clinical presentation may not help in diagnosing coexistence of both infections and, hence, radiology plays a major role in such cases.

Tuberculosis should always be suspected with radiological evidence of cavity consolidation ${ }^{8}$ or miliary mottling as seen in our cases. Typical hydatid disease presents as a cystic lesion more common in the lower lobes due to their better perfusion. ${ }^{7}$ The $\mathrm{CT}$ thorax is useful in delineating the size, extent, localization, calcification, communication with other organs, and for assessment of fluid density. In complicated hydatid cysts, a variety of radiological signs denoting different appearances of the cyst have been described. A ruptured cyst may present with classical findings on CT thorax. A double arch of air in the cyst forms the cumbo sign. ${ }^{9}$ In a completely collapsed cyst, the crumpled endocyst floats freely in the cyst fluid producing the water lily or Camelot sign. ${ }^{10,11}$ Ultrasonography may be useful in peripherally located lesions, and finding of doublelayered internal septum is considered highly specific for hydatid disease. ${ }^{12}$ If unruptured, such signs may be obtained postaspiration as noted in our case. Percutaneous aspiration of cyst was deferred until recently due to fear of postprocedure anaphylaxis; however, current evidence says that image-guided percutaneous diagnostic and therapeutic aspiration of hydatid cysts is associated with very low complication rates. ${ }^{13}$ Post-tap anaphylaxis was not seen in our cases too. Microbiological evidence of TB was present in both our cases, but the diagnosis of hydatid disease was predominantly based on radiological features. Both cases failed to show hydatid scolices. Serology in hydatid disease is often positive, but it has 20 to $30 \%$ false negative rates, and negative results are more likely in cases of unruptured or calcified cysts ${ }^{9}$ or unviable larvae. We could unearth only a few reports on coexistence of pulmonary hydatidosis and TB on an extensive literature review. The diagnosis essentially was made in these cases based on clinicoradiological and histopathological features. The microbiological evidence of tuberculous infection or parasitological 
evidence for hydatid was seldom obtained. Reports have also described the coexistence of these two infections in a single lesion. ${ }^{14}$

The management of hydatid disease is essentially surgical with complete chances of cure and negligible mortality rates in experienced hands. Lung-preserving surgery should be the preferred approach in all cases. ${ }^{15}$ However, surgery may be difficult in cases of complicated or disseminated hydatidosis, recurrent cysts, multiple cysts, in patients with multiorgan involvement, and inoperable cases. These cases are candidates for medical management ${ }^{15}$ with benzimidazoles group of drugs (mebendazole or albendazole, recommended doses being 40-50 and 10-15 mg/kg/day respectively). Albendazole is preferred because of its better bioavailability. The total duration of therapy is 3 to 6 months. The treatment for TB is a standard short-course chemotherapy consisting of a combination of antituberculous drugs under the Revised National Tuberculosis Control Programme. In cases with hydatid TB coinfection, surgical treatment for hydatid cyst should be deferred until antituberculous treatment is completed to decrease the peri- and postoperative morbidity and mortality. In our patients, upon reaching the diagnosis, simultaneous treatment of both diseases was instituted with ATT and Albendazole. Patients reported no significant adverse effects except for gastroesophageal reflux, which was controlled by proton pump inhibitor therapy. Coexistence of pulmonary TB and hydatid disease is rare, but not unknown. The diversity of the clinical presentations warrants the need for diagnosis by a multimodality approach with clinicoradiological, serological, and microbiological correlation. Our cases highlight the importance of suspicion of this coinfection in areas where TB and Echinococcosis are endemic, to enable early diagnosis and prevent morbidity. We suggest simultaneous medical therapy of both diseases in similar scenarios.

\section{REFERENCES}

1. Rao SS, Mehra B, Narang R. The spectrum of hydatid disease in rural central India: an 11-year experience. Ann Trop Med Public Health 2012 Jul;5(3):225-230.

2. Karande SC, Sheth SS, Lahiri KR, Shah MD. Coexistent hydatid disease and pulmonary tuberculosis in a five year old girl. J Assoc Physicians India 1991 Apr;39(4):353-354.

3. Chauhan MS, Rajan RS, Gopinathan VP, Jayaswal R. Pulmonary hydatid disease associated with pulmonary tuberculosis. Indian J Chest Dis Allied Sci 1986 Apr-Jun;28(2):88-91.

4. Li XX, Zhou XN. Co-infection of tuberculosis and parasitic diseases in humans: a systematic review. Parasit Vectors 2013 Mar; 6:79.

5. Santivanez S, Garcia HH. Pulmonary cystic echinococcosis. Curr Opin Pulm Med 2010 May;16(3):257-261.

6. Yang YR, Gray DJ, Ellis MK, Yang SK, Craig PS, McManus DP. Human cases of simultaneous echinococcosis and tuberculosis—significance and extent in China. Parasit Vectors 2009 Nov;2(1):53.

7. Beggs I. The radiology of hydatid disease. AJR Am J Roentgenol 1985 Sep;145(3):639-648.

8. Naidoo R. Active pulmonary tuberculosis: experience with resection in 106 cases. Asian Cardiovasc Thorac Ann 2007 Apr;15(2):134-138.

9. Morar R, Feldman C. Pulmonary echinococcosis. Eur Respir J 2003 Jun;21(6):1069-1077.

10. Kaur M, Singh R. Ruptured pulmonary hydatid cyst: the camalote sign. Indian J Clin Pract 2013 May;23(12):856-859.

11. Garg MK, Sharma M, Gulati A, Gorsi U, Aggarwal AN, Agarwal R, Khandelwal N. Imaging in pulmonary hydatid cysts. World J Radiol 2016 Jun;8(6):581-587.

12. Golzari SE, Sokouti M, Ghaffari A, Bazzazi AM, Ghabili K. Ultrasonography in diagnosis of pulmonary hydatid cysts. Lancet Infect Dis 2013 Apr;13(4):294.

13. Mawhorter S, Temeck B, Chang R, Pass H, Nash T. Nonsurgical therapy for pulmonary hydatid cyst disease. Chest 1997 Nov;112(5):1432-1436.

14. Saeed MY, Ahmed AH, Elhassan NB, Elhassan AM. Concomitant tuberculosis and hydatid cyst in a solitary pulmonary nodule of left lower lobe. BMJ Case Rep 2009 Sep;2009:1738.

15. Sarkar M, Pathania R, Jhobta A, Thakur BR, Chopra R. Cystic pulmonary hydatidosis. Lung India 2016 Mar-Apr;33(2): 179-191. 